\title{
Study on Group Velocity of Oceanic Internal Solitary Wave
}

\author{
X.D. Zhang \\ College of information science and engineering \\ Ocean University of China \\ Qingdao, Shandong, China \\ J. Wang \\ College of information science and engineering \\ Ocean University of China \\ Qingdao, Shandong, China
}

\author{
L.N. Sun \\ First Institute of Oceanography \\ State Oceanic Administration \\ Qingdao, Shandong, China \\ J.M. Meng \\ First Institute of Oceanography \\ State Oceanic Administration \\ Qingdao, Shandong, China
}

\begin{abstract}
Internal solitary wave is one of difficult problems in ocean field, scholars have applied different methods to investigate parameters of internal solitary wave, and group velocity is an important parameter for the internal solitary wave prediction. In this paper, equation used to describe group velocity of internal solitary wave was developed based on the Laplace equation and Bernoulli equation. Factors affecting the group velocity was also discussed, such as density difference of upper and lower layer, change of stratification, depth of water and wavelength of internal solitary wave. The results show that the group velocity became larger while the density difference and depth upper layer became bigger and the water became deeper, analysis also found that long-wavelength internal solitary wave travel faster that short-wavelength internal solitary wave. The calculation of group velocity in both South China Sea and Malin Shelf show good agreements with situ data, this means the equation is suitable for the description of group velocity of oceanic internal solitary wave.
\end{abstract}

Keywords-oceanic internal solitary wave; group velocity; Bernoulli equation

\section{INTRODUCTION}

Ocean internal solitary wave is a kind of wave which occurs in the density stratification sea water. The parameters of internal solitary wave such as wavelength and period change in a wide range, the wavelength range from less than 100 meter to tens of kilometer, period range from few minutes to dozens of hour, amplitude usually range from few meter to more than 100 meter[1]. According to in situ data the group velocity of internal solitary wave also has big variation, in deep sea the velocity of internal solitary wave is more than $3 \mathrm{~m} / \mathrm{s}$, while on the continental shelf the velocity is less than $1 \mathrm{~m} / \mathrm{s}[2,3]$. A moored array of sensors was deployed across the Chinese continental shelf and slop during the ASIAEX experiment in 2001[4], and phase speed of internal solitary wave in South China Sea is obtained with ADCP. Huang X et al.(2010)[5] conducted a long-term observation of internal solitary wave in South China Sea and they observed that the maximum speed of internal solitary wave propagate westward was $1.8 \mathrm{~m} / \mathrm{s}$. Not only the situ data, scholars also study the internal solitary wave velocity in theory, the most popular method is the phase speed obtained from KdV equation. Yi Zheng et al.(2013) [6]calculated the phase speed of internal solitary wave in South China Sea based on $\mathrm{KdV}$ equation.

In this paper, equation used to describe the group velocity of internal solitary wave is developed based on basic equations of fluid mechanics. The purpose is to study internal solitary wave propagation properties and hope to give some references for the prediction of internal solitary wave.

\section{MODEL DESCRIPTION}

For the incompressible, inviscid and irrotational twolayer fluid with the density of $\rho_{i}$, the fluid speed scalar potential $\phi_{i}$ and the pressure $p_{i}$ satisfy the following Laplace equation and Bernoulli equation[7]:

$$
\begin{gathered}
\varphi_{x x}+\varphi_{z z}=0, \eta \leq z \leq h_{1},-h_{2} \leq z \leq \eta \\
\varphi_{t}+\frac{1}{2}\left(\varphi_{x}^{2}+\varphi_{z}^{2}\right)+g \zeta=-\frac{p_{1}}{\rho_{l}}, z=\zeta
\end{gathered}
$$

Here, $\eta$ means the amplitude of internal solitary wave.

The boundary conditions at the upper and lower rigid surfaces are:

$$
\varphi_{z}=0, z=h_{1}, \quad z=-h_{2}
$$

As the fluid velocity and pressure at the interface are continuous, the boundary conditions of the two-layer fluid are given by

$$
\begin{gathered}
\zeta_{t}+\varphi_{x} \zeta_{x}=\varphi_{z}, z=\zeta \\
p_{1}=p_{2}, z=\zeta
\end{gathered}
$$

Two independent non-dimensional parameters are introduced:

$$
\varepsilon_{1}=\frac{A_{0}}{h_{1}}, \mu_{1}=k h_{1}
$$

Here, A0 means the initial amplitude of internal solitary wave, $\mathrm{k}$ means the wave number. $\varepsilon$ and $\mu$ are two small parameters: $O\left(\varepsilon_{1}\right)=O(l), \mu_{1} \ll 1$. $\varepsilon$ means the relations between amplitude and depth of upper layer, $O\left(\varepsilon_{1}\right)=O(l)$ 
means this relation satisfy the condition of large amplitude internal solitary wave.

When $\eta \leq z \leq h_{1}$, we focus on the upper fluid $(i=1)$ first, non-dimensional form of the upper fluid equations $(1) \sim(4)$ are:

$$
\begin{gathered}
\mu^{2} \varphi_{x x}+\varphi_{z z}=0, \eta \leq z \leq 1 \\
\mu^{2}\left(\varphi_{t}+\eta\right)+\frac{1}{2}\left[\mu^{2} \varphi_{x}^{2}+\varphi_{z}^{2}\right]=-\mu^{2} p_{1}, z=\eta \\
\varphi_{z}=0, z=\eta \\
\mu^{2}\left(\eta_{t}+\varphi_{x} \eta_{x}\right)=\varphi_{z}, z=1
\end{gathered}
$$

Introduce Taylor expansion and combining equations (7) (9) we can get:

$$
(\eta-1)^{2}\left(-p_{1}\right)=-c_{0}^{2} \eta(\eta-1)+\eta(\eta-1)^{2}+\frac{1}{2} c_{0}^{2} \eta^{2}
$$

Dimensional forms of equation (10) is

$$
\left(\frac{\eta}{h_{l}}-1\right)^{2} \frac{-p_{l}}{\rho_{l}}=\left(g+\frac{c_{0}^{2}}{h_{l}}\right) \eta+\left(\frac{k^{2}}{2} c_{0}^{2}-\frac{c_{0}^{2}}{2 h_{l}}-\frac{2 g}{h_{l}}\right) \eta^{2}+\frac{g}{h_{l}^{2}} \eta^{3}(11)
$$

For the lower fluid $(i=2)$, the small parameters are $\varepsilon_{2}=\frac{A_{0}}{h_{2}}, \mu_{2}=k h_{2}$. Here we have $O\left(\mu_{2}\right)=O(l), \varepsilon_{2}<<1$, for $O\left(\mu_{2}\right)=O(l)$ means the wavelength of internal solitary wave is comparable with depth of water, this also means this model is suitable for deep-sea internal solitary wave. When $-h_{2} \leq z \leq \eta$, we have

$$
\begin{gathered}
\varphi_{x x}+\varphi_{z z}=0,-h_{2} \leq z \leq \zeta(12) \\
\varphi_{t}+\frac{1}{2}\left(\varphi_{x}^{2}+\varphi_{z}^{2}\right)=-g \zeta-\frac{p_{2}}{\rho_{2}}, z=\zeta \\
\zeta_{t}+\varphi_{x} \zeta_{x}=\varphi_{z}, z=\zeta(14) \\
\varphi_{z}=0, z=-h_{2}(15)
\end{gathered}
$$

Combining equation (5) (11)(13) we got

$$
\begin{gathered}
{\left[\varphi_{t}+\frac{1}{2}\left(\varphi_{x}^{2}+\varphi_{z}^{2}\right)\right]\left[\left(\frac{\eta}{h_{1}}-1\right)^{2}\right]=-c_{1} \eta+c_{2} \eta^{2}+c_{3} \eta^{3},} \\
c_{1}=-\frac{\left(\rho_{1}-\rho_{2}\right) g h_{1}+\rho_{1} c_{0}^{2}}{\rho_{2} h_{1}} \\
c_{2}=\frac{-\rho_{1} c_{0}^{2}+4\left(\rho_{2}-\rho_{1}\right) g h_{1}}{2 \rho_{2} h_{1}^{2}} \\
c_{3}=\frac{\left(\rho_{1}-\rho_{2}\right) g}{\rho_{2} h_{1}^{2}} .
\end{gathered}
$$

Differentiating (16) over $\mathrm{t}, \mathrm{x}, \mathrm{z}$, three equations could be get. Combine them to one and seek series expansion of it in power of $\eta$ at z=0:

$$
\begin{aligned}
& {\left[\varphi_{t t}+\varphi_{t z} \eta+\frac{1}{2} \varphi_{t z z} \eta^{2}+\left(\varphi_{x}^{2}+\varphi_{z}^{2}\right)_{t}+\left(\varphi_{x}^{2}+\varphi_{z}^{2}\right)_{t z} \eta+\frac{1}{2} \varphi_{x}\left(\varphi_{x}^{2}+\varphi_{z}^{2}\right)_{x}+\frac{1}{2} \varphi_{z}\left(\varphi_{x}^{2}+\varphi_{z}^{2}\right)_{z}\right]} \\
& {\left[\left(\frac{\eta}{h_{1}}-1\right)^{2}\right]+\left[\varphi_{t}+\varphi_{t z} \eta+\frac{1}{2} \varphi_{t z z} \eta^{2}+\frac{1}{2}\left(\varphi_{x}^{2}+\varphi_{z}^{2}\right)+\frac{1}{2}\left(\varphi_{x}^{2}+\varphi_{z}^{2}\right)_{z} \eta\right]\left[\frac{2}{h_{1}}\left(\frac{\eta}{h_{1}}-1\right)\left(\varphi_{z}+\varphi_{z z} \eta\right)\right]} \\
& =-c_{1} \varphi_{z}-c_{1} \varphi_{z z} \eta-\frac{1}{2} c_{l} \varphi_{z z z} \eta^{2}+2 c_{2} \varphi_{z} \eta+2 c_{2} \varphi_{z z} \eta^{2}+3 c_{3} \eta^{2} \varphi_{z} .
\end{aligned}
$$

Similarly, the series expansion of equation (16) is :

$$
-c_{1} \eta=\left[\varphi_{t}+\varphi_{t z} \eta+\frac{1}{2} \varphi_{t z} \eta^{2}+\frac{1}{2}\left(\varphi_{x}^{2}+\varphi_{z}^{2}\right)+\frac{1}{2}\left(\varphi_{x}^{2}+\varphi_{z}^{2}\right)_{z} \eta\right]\left[\left(\frac{\eta}{h_{l}}-1\right)^{2}\right]-c_{2} \eta^{2}-c_{3} \eta^{3}
$$

When apply the multiple-scale method [8] to equation (12) (17) (18), we get:

$$
\begin{gathered}
\left(\frac{\partial^{2}}{\partial x_{0}^{2}}+\frac{\partial^{2}}{\partial z^{2}}\right) \varphi_{n}=F_{n},\left(-h_{2} \leq z \leq \zeta\right) \\
\left(c_{1} \frac{\partial}{\partial z}+\frac{\partial^{2}}{\partial t_{0}^{2}}\right) \varphi_{n}=G_{n},(z=\zeta) \\
-c_{1} \eta_{n}=H_{n},(z=\zeta)
\end{gathered}
$$

$F_{n} 、 G_{n} 、 H_{n}$ means the sum of all the items except items on the left side.

Having in mind the slow evolution of a wave train, we develop $\phi \mathrm{n}$ as follows[9]:

$$
\varphi_{n}=\sum_{m=0}^{n} \varphi_{n m} e^{i m \psi}+c . c . \quad(\mathrm{m}=0,1,2,3,4 \ldots)
$$

Where $\psi=k x-\omega t$ is phase, c.c. stands for conjugate complex number, $\phi_{n m}$ is the functions of the slow variables and $\mathrm{z}$.

The boundary value problems for each harmonic $(\mathrm{m})$ of each order ( $n$ ) with the variable $Z$ can be obtained by substituting equation above into the equations (19) (21) and (15).

$$
\begin{gathered}
\left(\frac{\partial^{2}}{\partial z^{2}}-m^{2} k^{2}\right) \varphi_{n m}=F_{n m} \quad-h_{2} \leq z \leq \eta(22) \\
\left(c_{1} \frac{\partial}{\partial z}-m^{2} \omega^{2}\right) \varphi_{n m}=G_{n m} \quad z=\eta(23) \\
\eta_{n m}=-\frac{1}{c_{1}} H_{n m} \quad z=\eta(24) \\
\left(\varphi_{n m}\right)_{z}=0 \quad z=-h_{2}(25)
\end{gathered}
$$

Solve the equations above, when $n=1$ we can get the equation which described the group velocity of internal solitary wave.

$$
\begin{gathered}
F_{10}=0, F_{11}=0 ; \\
G_{10}=0, G_{11}=0 ; \\
H_{10}=0, H_{11}=-i \omega \varphi_{11} . \\
\text { When } \eta_{1}=\frac{1}{2}\left(A e^{i \psi}+\text { c.c. }\right), \text { combine }(22) \sim(25) \text { we get } \\
\omega^{2}=c_{1} k t h\left(k h_{2}\right)(26)
\end{gathered}
$$

So the equation of group velocity is

$$
c_{g}=\frac{d \omega}{d k}=\frac{\omega}{2 k}\left[1+\frac{2 k h_{2}}{\operatorname{sh}\left(2 k h_{2}\right)}\right]
$$

From the equation above, we can find that many factors contribute to the group velocity. For example, stratification of the ocean will change along with the seasonal variation, and group velocity will change due to the change of stratification. Some other factors such as, wavelength of 
internal solitary wave and depth of water will also affect the velocity.

\section{CALCUlation AND DiscUSSION OF GROUP VELOCITY}

In this part, we discussed how the group velocity will change with different factors and comparison with the situ data are given.

\section{A. Factors Affecting Group Velocity}

To applied the theory in practice, a special site on $\left(120^{\circ}\right.$ E, $21^{\circ} \mathrm{N}$ ) was chosen to obtain the parameter needed for the calculation of group velocity. Data was downloaded from Etop1 and CISL Research Data Archive. Depth of water on this site is about $3300 \mathrm{~m}$, based on the real hydrological parameters, four cases were studied to understand the trend of the speed: density difference, different stratification, different depth of water and different wavelength of internal solitary wave. Assuming the ocean is a two-layer model, h1 means the depth of upper layer and h2 means the depth of lower layer. Lamda stands for the wavelength of internal solitary wave ranging from few meter to tens of kilometer.

The results of the numerical simulation and calculation as follows.

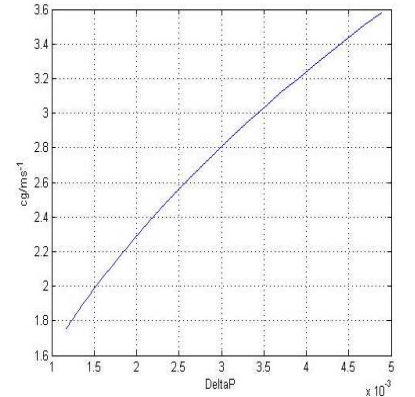

(a)

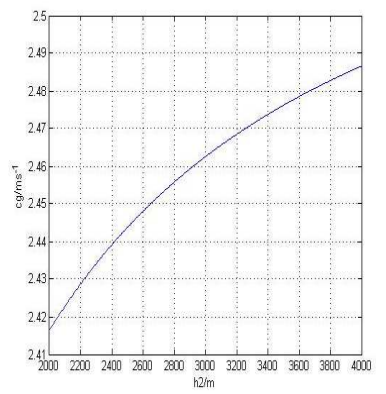

(c)

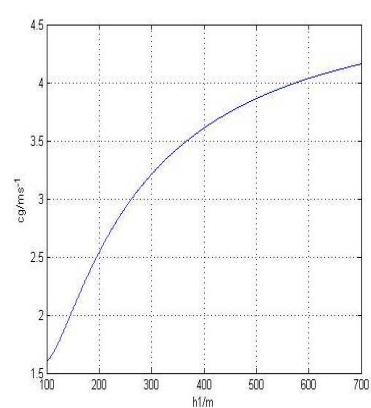

(b)

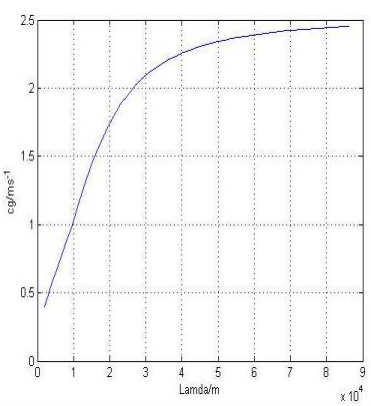

(d)
FIGURE I. GROUP VELOCITY VARIES FOR DIFFERENT FACTORS

(A)DIFFERENT DENSITY DIFFERENCE OF UPPER AND LOWER

LAYER;(B)DIFFERENT STRATIFICATION;(C)DIFFERENT DEPTH OF WATER;(D)DIFFERENT WAVELENGTH OF INTERNAL SOLITARY WAVE.

Based on Figure 3.1 some conclusions could be drawn. From (a) we can find that when the relative density difference $\left(\frac{\rho_{2}-\rho_{1}}{\bar{\rho}}\right)$ between the upper and lower layer became larger, group velocity will also become larger. (b)shows that group velocity of internal solitary wave became larger with the increase of depth of upper layer, usually depth of upper layer in deep sea is bigger than that in shallow water. (c)shows when the upper layer keep the same, with the increase of total depth of water, the group velocity shows the trend of increase. To some extent, this can explain why internal solitary wave travel faster in deep sea. From (d) we can find that internal solitary wave with larger wavelength will travel faster than those with relatively short wavelength. Here, the wavelength range from few kilometer to tens of kilometer due to site B2 is in deep sea area, the wavelength in deep sea is relatively large. We can also find that although the group velocity of internal solitary wave grows faster on the initial period, the larger the wavelength the smaller the increase.

\section{B. Calculation of Group Velocity}

On the basis of the variation trend of group velocity discussed above, combined with the abundant field study of the oceanic internal solitary wave. Four sites were chosen to calculate the group velocity of internal solitary wave. The first site is from Chang M H et.al(2011)[10], ADCP was applied to measure the speed, LR1 with the water depth about $610 \mathrm{~m}$ was chosen in their study, marked as A in table 4.1. The second site was on Malin Shelf [11], depth of water is about $720 \mathrm{~m}$,marked as B in table 4.1. The third site is around Dongsha Atoll marked as $\mathrm{C}$ in table 4.1,depth of water at C is from 190m 300m. Ke-Hsien Fu et.al(2012) [3]conducted a field study of internal solitary wave and data of internal solitary wave speed was obtained. The fourth site marked as D in table 4.1 is from remote sensing data, Xiaodong Huang et.al(2012)[12] get the data through MODIS image,depth of water here is about $500 \mathrm{~m}$. Data for all the four sites were downloaded and processed, and calculation is done based on equation (27), the results of comparison are shown in table 1 .

\section{TABLE I. COMPARISON BETWEEN SITU DATA AND} CALCULATION DATA OF GROUP VELOCITY.

\begin{tabular}{|l|c|c|c|c|}
\hline & A & B & C & D \\
\hline Situ data & $2 \sim 2.5 \mathrm{~m} / \mathrm{s}$ & $57 \pm 9 \mathrm{~cm} / \mathrm{s}$ & $\begin{array}{c}0.74 \sim 1.05 \\
\mathrm{~m} / \mathrm{s}\end{array}$ & $\begin{array}{c}1.26 \sim 1.72 \\
\mathrm{~m} / \mathrm{s}\end{array}$ \\
\hline $\begin{array}{l}\text { Calculation } \\
\text { data }\end{array}$ & $1.95 \mathrm{~m} / \mathrm{s}$ & $50.2 \mathrm{~cm} / \mathrm{s}$ & $\begin{array}{c}0.69 \sim 0.82 \\
\mathrm{~m} / \mathrm{s}\end{array}$ & $1.55 \mathrm{~m} / \mathrm{s}$ \\
\hline
\end{tabular}

Table 1 shows good agreement between situ data and calculation in theory. Site A was the phase speed obtained from $\mathrm{ADCP}$, calculation data was group velocity developed based on the dispersion relation, usually the group velocity is smaller than the phase speed. The result shows that the equation (27) is suitable for the description of group velocity of internal solitary wave. The difference between the situ data and calculation data may due to the following reasons: (1) oceanic parameter varies because of the seasonal variation; (2)different wavelength of internal solitary wave leads to different group velocity, the wavelength difference between the field study and the calculation; (3)influential ocean phenomena, such as Kuroshio Intrusion.

\section{CONCLUSION}

In this paper, based on basic equations of fluid mechanics, equation used to describe the group velocity of internal solitary wave is developed. Factors affecting the group 
velocity were discussed and the variation trend of group velocity was also given. The result shows that with larger density difference, depth of upper layer and total water depth, the group velocity has an increase trend. internal solitary wave with larger wavelength will travel faster than those with relatively short wavelength, and the larger the wavelength the smaller the increase. Comparison between the situ data and the calculation data was given, the results show a good agreement. So, the equation is suitable for the description of internal solitary wave and undoubtedly will give some reference for the prediction of internal solitary wave.

\section{ACKNOWLEDGMENTS}

We would like to thank financial supports from the Natural Science Foundation of China (No. 61171161).

\section{REFERENCE}

[1] Fang Xinhua, Du Tao. Fundamentals of oceanic internal waves and internal waves in the China Sea. Qingdao:China Ocean University Press, 2005,1-4

[2] Wei Zhao, Xiaodong Huang, Jiwei Tian. A new method to estimate phase speed and vertical velocityof internal solitary waves in the South China Sea. J Oceanogr (2012) 68:761-769

[3] Ke-Hsien Fu, Yu-Huai Wang, Louis St. Laurent, Harper Simmons, Dong-Ping Wang.Shoaling of large-amplitude nonlinear internal waves at Dongsha Atoll in the northern South China Sea. Continental Shelf Research 37 (2012) 1-7

[4] Ramp S R, Tang T Y, Duda T F, et al. Internal solitons in the northeastern South China Sea. Part I: Sources and deep water propagation[J]. Oceanic Engineering, IEEE Journal of, 2004, 29(4): 1157-1181.

[5] Huang X, Zhao W, Tian J, et al. Mooring observations of internal solitary waves in the deep basin west of Luzon Strait[J]. Acta Oceanologica Sinica, 1-8.

[6] Yi Zheng, Daqi Xin, Shuxia Li, et al.Current field features and propagation characteristics of suspected internal solitary wave packet. Ocean Engineering, 72(2013)448-452

[7] Qiangzhong Mei. Dynamic of water wave. Beijing: science press, 1984.331

[8] Cole. J. D. Perturbation Methods in Applied Mathematics. Blaisdell. Waltham. Mass, 1968

[9] J. P. Boyd. Equatorial solitary waves, Part II :envelope solitons, J. Phys. Oceangr. 1983, 13:428 449

[10] Chang M H, Lien R C, Yang Y J, et al. Nonlinear Internal Wave Properties Estimated with Moored ADCP Measurements[J]. Journal of Atmospheric \& Oceanic Technology, 2011, 28(6).

[11] Small, J., Z. Hallock, G.. Pavey, J. Seott. Observations of Large Amplitude lntemal Waves at the Malin Shelf Edged during SESAME 1995, Continental Shelf Res., 1999, 19: 1389 1436

[12] Huang X D, Tian J W, Zhao W. The Behaviors of Internal Solitary Waves near the Continental Shelf of South China Sea Inferred from Satellite Images[J]. Advanced Materials Research, 2012, 588: 21312135 